\title{
Wernicke's Encephalopathy with Generalized Weakness In a Woman with Hyperemesis Gravidarum and Gestational Thyrotoxicosis: A Case Report of a Rare Co-Occurrence with Literature Review
}

\author{
Parul Dubey ${ }^{1}$, Sachin Narvekar ${ }^{2}$, Deepa S.Nadkarni ${ }^{3}$ and Shradha Naik ${ }^{4}$ \\ ${ }^{1,4}$ Department of Neurology, Goa Medical College, Bambolim, Goa, India \\ ${ }^{2}$ Gynecologist, Department of Obstetrics \& Gynecology \\ Manipal Hospitals Goa Dona Paula, Goa, India \\ ${ }^{3}$ Radiologist, Radiance Diagnostics, Dona Paula,Goa, India
}

Correspondence should be addressed to: Parul Dubey;

Received date: 13 July 2015; Accepted date: 21 October 2015; Published date: 24 February 2016

Academic Editor: Andrzej Szutowicz

Copyright (C) 2016. Parul Dubey, Sachin Narvekar, Deepa S.Nadkarni and Shradha Naik. Distributed under Creative Commons CC-BY 4.0

\begin{abstract}
Wernicke's encephalopathy(WE) is an uncommon, yet, treatable and acute neuropsychiatric manifestation of thiamine deficiency which may often go undiagnosed .We report this case of a previously healthy 30 year old, non drinker, pregnant woman with hyperemesis gravidarum (HG) and gestational thyrotoxicosis(GT) who presented with a combination of WE, polyneuropathy and dysautonomia. This case is unique in many respects. (WE) is a rare but serious known complication of hyperemesis gravidarum. More so, WE occurring in association with hyperemesis gravidarum and gestational thyrotoxicosis, which are both hypermetabolic states and may predispose to $\mathrm{WE}$, has been rarely reported in literature. Association of polyneuropathy causing generalized weakness and autonomic dysfunction in the same patient as part of the thiamine deficient state, with a good treatment outcome further adds to the complex yet interesting clinical presentation of this case.
\end{abstract}

Keywords: Wernicke's encephalopathy,hyperemesis gravidarum,gestational thyrotoxicosis,polyneuropathy.

Cite this Article as: Parul Dubey , Sachin Narvekar, Deepa S.Nadkarni and Shradha Naik (2016)," Wernicke's Encephalopathy with Generalized Weakness In a Woman with Hyperemesis Gravidarum and Gestational Thyrotoxicosis: A Case Report of A Rare Co-Occurrence with Literature Review", International Journal of Case Reports in Medicine, Vol. 2016 (2016), Article ID 376298, DOI: 10.5171/2016.376298 


\section{Introduction}

Wernicke's encephalopathy is an acute neuropsychiatric condition which occurs due to thiamine deficiency in various catabolic states and can often go overlooked. A high index of suspicion for the condition in suggestive clinical settings is important as rapid reversal of symptoms is possible with treatment and if left untreated it may progress to permanent neurological disability.

\section{Case Report}

A 30 year old Indian lady primigravida at 16 weeks gestation, belonging to a low socioeconomic class, presented to the casualty of our hospital on Oct 20th 2013, with history of acute onset difficulty in walking and weakness of all four limbs for six days, headache for two days and double vision and severe breathlessness for last one day.

She did not have any decreased vision, eyelid drooping, facial numbness or deviation, vertigo, decreased hearing, ringing in ears, change in speech, difficulty in swallowing, unilateral limb weakness, tingling, burning sensation, numbness or any bladder bowel disturbances. There were no associated seizures, loss of consciousness, back or neck pain.

For the past one and a half month she had excessive nausea and repeated vomitings and was not eating well. She was hospitalized elsewhere about 15 days back for recurrent vomitings and intravenous fluids and antiemetics were administered. Since then, vomitings were controlled.

During her routine pregnancy check up she was also found to have hyperthyroidism. There were no other known associated medical illnesses. She had lost about $5 \mathrm{~kg}$ of weight in the past 2-3 months.

There was no history of any prior or associated fever, cough, loose motions, joint pains or swelling, skin rashes, body aches or abdominal pain or ingestion of any canned foods ,shell fish, any drug or toxin, snake bite or dog bite any time prior to the illness.

On examination, her pulse rate was 130 per minute; blood pressure was $100 / 60 \mathrm{~mm} \mathrm{Hg}$. She was breathless and her respiratory rate was 40 per minute and temperature was $99 \mathrm{oF}$. She was very thin, weighed $36 \mathrm{~kg}$ and was pale but there was no clinical evidence of jaundice, clubbing, cyanosis, lymphadenopathy, edema or goiter but had bulging eyes.

She was conscious but disoriented to place and time. She had difficulty in speaking because of breathlessness but had no apparent nasal twang in speech. Pupils were of normal size reacting normally to light, bilateral horizontal gaze paresis and bilateral horizontal gaze evoked jerky nystagmus were present. Vertical eye movements were normal. Fundus examination revealed no papilloedema. Other cranial nerve examination was also normal.

There was generalized hypotonia, severe weakness of neck, trunck and all four limbs (MRC grade 2/5). She was bedbound, unable to even sit up unsupported in bed .All deep tendon reflexes were absent. Plantar responses were bilateral flexor. Abdominal reflexes were present. Limb ataxia could not be assessed because of marked weakness of all four limbs. Sensory examination was completely normal. There were no signs of meningeal irritation.

Chest auscultation revealed basal crepitations bilaterally. Per abdominal and cardiovascular examination were normal. Gynecological assessment revealed a 16 weeks gravid uterus.

Considering the acute onset of weakness of all four limbs, neck and truncal weakness with headache, horizontal gaze paresis, nystagmus with respiratory involvement, possibilities of Miller Fisher variant of Guillain Barre Syndrome, acute disseminated 
encephalomyelitis, brainstem lesions such as demyelination, pontine myelinolysis or space occupying lesions, acute myasthenic crisis or metabolic causes like electrolyte disturbances were initially considered. Also in view of a hypermetabolic state as suggested by the history of HG and hyperthyroidism, a possibility of WE was considered. CT Brain, plain and contrast was normal.

Supportive management was started in the casualty and injection thiamine in high dose (500 mg in normal saline, three times a day) was immediately started after sending blood samples for thiamine level.ABG revealed hypoxia. As the patient was in respiratory distress she was immediately put on artificial ventilation in the medical ICU. Electrocardiogram showed sinus tachycardia and chest roentgenogram was normal. No evidence of cardiomegaly or pneumonia were found. Blood reports came back as detailed in Table 1.She had anemia, hypokalemia and hypoalbuminemia.

Central line was passed and she was simultaneously initiated on intravenous fluids, potassium correction, vitamin B12 1500 micro grams daily, intravenous antibiotics, folvite and iron supplementation and low molecular weight heparin for prophylaxis for deep venous thrombosis.

Thyroid profile suggested hyperthyroidism.HIV ELISA, Hbs Ag, Anti HCV, ANA, ACE, Anti TPO antibodies were negative. Serum CPK was 60IU/L. Serum lactate was $10 \mathrm{mg} / \mathrm{dL}$ (Normal 9-16 $\mathrm{mg} / \mathrm{dL}$ ).Cerebrospinal fluid examination showed normal sugar and protein and no cells. A 2D echocardiogram was also normal with a normal left ventricular ejection fraction .USG abdomen showed a single live fetus of 16 weeks gestational age.

A plain MRI brain done the next day revealed symmetrical areas of altered signal intensity in the periaqueductal grey matter, tectal plate, both thalami, dorsal brainstem and in the mamillary bodies ,highly suggestive of
Wernicke's encephalopathy (Figure 1 \&2) .MRI of the spine was normal.

On the basis of the brain MRI findings, the differential diagnosis was narrowed down and pointed strongly in favour of Wernicke's encephalopathy with associated generalized weakness either due to neuropathy, most probably secondary to nutritional deficiency of thiamine and other B complex vitamins or hypokalemia or a combination of both.

Her serum thiamine levels came low 0.025 $\mu \mathrm{g} / \mathrm{dL}(0.2-2 \mu \mathrm{g} / \mathrm{dL})$. She was also started on propylthiouracil $50 \mathrm{mg}$ three times a day and beta blockers, after consultation with endocrinologist.

During the next few days she had significant daily fluctuation in heart rate from 50-140 bpm but maintained blood pressure without any ionotropic support. She was tracheostomised at the end of ten days of mechanical ventilation. She continued to be on ventilator for the next two months. During the course of ventilation she developed ventilator associated pneumonia.

After about a week of thiamine administration ophthalmoparesis started improving, by the end of one and a half month she was moving her eyes completely in all directions, though nystagmus persisted for a longer time and took another month to recover. Injectable thiamine was continued at a high dose of $500 \mathrm{mg}$ three times a day for one month and later on she was started on oral thiamine $100 \mathrm{mg}$ daily. Power of all four limbs gradually improved in the ICU to MRC grade $4 / 5$ in all four limbs at the end of three months of hospitalization. Physiotherapy of limbs and chest was carried out regularly.

She continued to be in the ICU due to hospital acquired pneumonia and septicemia and was being treated with multiple antibiotics according to the culture sensitivity results.

After a month propylthiouracil was replaced with neomercazole $10 \mathrm{mg}$ three times a day on the advice of the endocrinologist. The 
family was explained about the risk to the fetus because of exposure to multiple drugs and radiation due to repeated $\mathrm{X}$ rays. The mother and the family wished to continue with the pregnancy despite all these concerns. As the abdominal girth of the patient was increasing due to advancing pregnancy, weaning her from ventilator was not possible.

She started feeling the fetal movements around the fifth month of gestation. At six months of gestation, while still in the ICU, she started having lower abdominal pain and vaginal bleeding. Gynecologist decided for a lower segment caesarean section and a live male baby of 900 grams was delivered, who was admitted in the neonatal ICU. The baby was fed on expressed breast milk and formula feeds through the nasogastric tube. Over the next 2 months the baby gained weight and was discharged from the neonatal ICU. The baby weighed $1.5 \mathrm{~kg}$ at discharge.

After the delivery, the ventilatory requirements of the patient improved and weaning was facilitated. Over the next fifteen days we could wean her from the ventilator. She was shifted to the ward and was able to walk unassisted. Mild recent memory impairment was observed on later assessment in the ward.

A nerve conduction study could not be done initially because of the critical condition of the patient and the necessity to shift her to the ICU for mechanical ventilation. After discharge from ICU, however, a nerve conduction study of all four limbs was normal. Her repeat thyroid profile postpartum showed was normal. The lady was discharged home on 10th of March 2014 with her baby who was developing normally.

On 23 rd of September 2014 this lady came for her last follow up.She does not have any neurological deficits except mild memory deficits but independently managing her life activities and her child who is 6 months old is developing normally.

\section{Discussion}

Thiamine (vitamin $B_{1)}$, an essential nutrient, is an important cofactor for many enzymes involved in the Kreb's cycle and pentose phosphate pathway. Thiamine deficiency renders these enzymes inactive and pyruvate cannot enter the Kreb's cycle, which leads to energy deprivation in the tissues and excess accumulation of lactate and glutamate causing injury to specific regions of the brain with a high metabolic requirement, leading to the neuropsychiatric syndrome of "WE". This cell injury is initially reversible but if missed early on, often leads to irreversible damage to the neurons. Therefore, the great clinical significance of a high index of suspicion and a prompt and early institution of parenteral thiamine administration.

Thiamine deficiency may not only affect the nervous system but also the cardiovascular and gastrointestinal systems. Dry beriberi presents as an acute polyneuropathy and increased susceptibility to infections. Wet beriberi presents with generalized oedema, high cardiac output cardiac failure, vasodilatation or lactic acidosis (Shoshin beri beri).

Thiamine deficiency is more often encountered in alcoholics but even in non alcoholics; malignancy, AIDS, malnutrition, malabsorption, unbalanced diet, hemodialysis, renal diseases, prolonged total parenteral nutrition, bariatric surgery, iatrogenic glucose loading may cause WE. Hyperemesis gravidarum (HG) also predisposes for WE. Less commonly psychiatric disorders, bone marrow transplantation, intoxications and thyroid diseases have also been reported as predisposing factors for "WE".

Our case had WE which is a rare complication of HG per se. More so, WE occurring in association with HG along with GT, which are both catabolic states and may predispose to $\mathrm{WE}$, is a very rarely reported combination in literature. Association of generalized weakness and dysautonomia in

Parul Dubey, Sachin Narvekar, Deepa S.Nadkarni and Shradha Naik (2016), International Journal of Case Reports in Medicine, DOI: 10.5171/2016.376298 
the same patient with proven WE, as part of the thiamine deficient state is also a rare cooccurrence.

Our patient had a hyperthyroid state and also had clinical features suggesting thyrotoxicosis in the form of tachycardia, bulging eyes, muscle weakness etc. A transient mild hyperthyroid state is known to occur with $\mathrm{HG}$ and is seen in $60 \%$ of the cases, which resolves by 18 weeks of gestation without treatment or sequelae. Clinical thyrotoxicosis, as was seen in our case, is only found in a small proportion. These patients do not have a prior thyroid illness or goitre and thyroid antibodies are negative. This hyperthyroid state is attributable to a physiological increase in thyroxine binding globulin, total triiodothyronine and thyroxine concentrations, thyroglobulin and renal iodide clearance in pregnancy; a thyroid stimulating effect of hCG (which is high in HG); hCG hypersensitive thyrotrophin receptors in an overactive thyroid or probable secretion of more potent variant of hCG.

"WE" is classically described as a triad of clinical manifestations: global confusion, ophthalmoplegia and gait ataxia. The classical triad is seen only in $10 \%$ of cases and the absence of this triad does not rule out WE. Encephalopathy may manifest as frontal lobe dysfunction (apathy), agitation, confusion, seizures, mild memory impairment or coma. The most common ocular abnormality in patients with "WE" is a horizontal nystagmus. Complete ophthalmoplegia is seen only in more advanced disease. Unsteadiness of gait can also be mild to very severe. Other features of thiamine deficiency may accompany "WE" for eg. hypotension, hypothermia, vestibular dysfunction, polyneuropathy, high output cardiac failure ,gastrointestinal complaints like abdominal pain, nausea, vomitings and lactic acidosis. In our case at the time of presentation, apart from WE, generalized weakness, tachycardia, dyspnoea were also present which could be attributable to the cumulative effects of nutritional deficiencies (other B vitamins, Vitamin C, minerals), hyperthyroidism, septicemia and hypokalemia.

However, persistence of fluctuation in heart rate and quadriparesis throughout her ICU stay despite a successful correction of electrolytes and thyroid status; and gradual recovery over the next few months; would favour a dysautonomia and polyneuropathy secondary to thiamine deficiency.

WE with dysautonomia and polyneuropathy in the same patient is an uncommonly reported scenario in literature. Cognat et al have described severe dysautonomia in an HIV positive woman with WE and Wang etal have described a case of refractory hypotension after total gastrectomy which responded to thiamine administration. Bilateral lesions of thalami may be responsible for the autonomic dysfunction.

Our patient had a prolonged quadriparesis with muscle atrophy and hyporeflexia which improved gradually. We attribute this to the thiamine deficiency as it occurred in association with the "WE". Polyneuropathy has been described uncommonly with "WE" which is reversible with thiamine supplementation. The proposed mechanism of thiamine deficiency related polyneuropathy is an axonal dysfunction, possibly caused by decreased $\mathrm{Na}+-\mathrm{K}+-$ ATPase activity due to thiamine deficiency, which reverses on thiamine supplementation leading to a rapid clinical and electrophysiological recovery.

CT scan is not reliable for the diagnosis of WE and it was normal in our case too.MRI brain is superior in diagnosing WE. The brain structures most often involved are those surrounding the third ventricle, aqueduct and fourth ventricle in a symmetrical distribution.80\% of the cases have mammillary body involvement. Other structures commonly involved are dorsomedial thalami, ocular motor nuclei, vestibular nuclei, locus ceruleus and 
periaqueductal grey matter. Less commonly colliculi, fornices, septal regions, hippocampus and cerebral cortex may be involved.

A blood sample for thiamine level should be collected immediately before administration of thiamine. A normal thiamine level does not rule out the diagnosis of WE. Parenteral thiamine (intravenous) is the treatment of choice as oral thiamine is not effective. Although there is no consensus as to the dose requirement and duration for the treatment of WE, usually high doses $200 \mathrm{mg}$ (non alcoholics)or $500 \mathrm{mg}$ (alcoholics)three times a day are administered. Thiamine should be continued as long as signs and symptoms continue to improve.

In WE complicated with $\mathrm{HG}$, preterm delivery, intrauterine growth retardation and miscarriage may occur. Spontaneous foetal loss rate is about $37 \%$, elective abortion rate is about $10 \%$.Our case too had a preterm delivery but her baby survived and is developing normally. Effective thiamine therapy may improve chances of delivering a healthy newborn.

Thiamine replacement leads to a complete recovery in 20\%.Ataxia tends to recover in $50 \%$ but retentive memory deficits generally persist. After thiamine supplementation ophthalmoplegia usually begins to improve within hours.15-20\% of the WE patients may die of infections or liver failure. Our case too suffered from serious infections and had a preterm delivery during the course of hospitalization, but finally she had a good treatment outcome and was discharged without severe neurological deficits.

The initial diagnosis of WE at casualty level is always clinical. Its a good clinical clue to consider WE in cases presenting with two of the following four conditions: nutritional deficiency, ocular findings, cerebellar dysfunction (ataxia) and mental status changes. It is a good clinical practice to supplement parenteral thiamine whenever there is the slightest doubt of a predisposing situation for $\mathrm{WE}$, as one may avert severe neuronal damage
To conclude, we report this case of WE with multiple other clinical manifestations of thiamine deficiency, in a young pregnant woman with HG and GT, not only because of its rarity but also to highlight the clinical importance of a high index of suspicion and a rapid thiamine administration in the appropriate settings, which may lead to a good neurological outcome.

\section{References}

1. Antunez E,Estruch R,Cardenal C,Nicolas JM,Fernandez-Sola J,Urbano Marquez A.Usefulness of CT and MR imaging in the diagnosis of acute wernicke's encephalopathy.AJR Am $\quad J \quad$ Roentgenol 1998;171:1131-1137.

2. Butterworth RF,Kril JJ,Harpe CGR.Thiamine dependent enzyme changes in the brains of alcoholics:relationship to the Wernicke-Korsakoff syndrome Alcohol Clin Exp Res.1993;17:1084-1088.

3. Caine D,Halliday GM,Kril JJ etal.Operational criteria for the classification of chronic alcoholics:identification of wernicke's encephalopathy.J Neurol Neurosurg Psychiatry.1997;62:51-60

4. Chiossi G, Neri I, Cavazzuti M, Basso G, Facchinetti F. Hyperemesis gravidarum complicated by Wernicke encephalopathy: background, case report, and review of the literature. Obstet Gynecol Surv 2006; 61: 25568.

5. Cognat E, Hainque E, Mesnage V, Levy R. Severe dysautonomia revealing Wernicke's encephalopathy. Rev Neurol (Paris.) 2011;167:250-53.

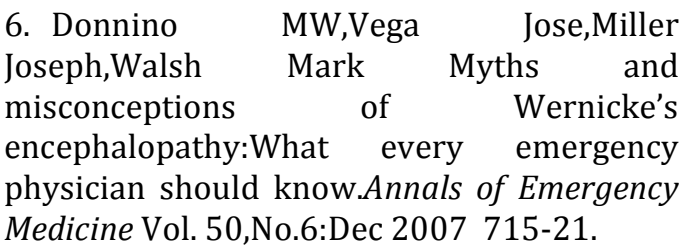


7. Fattal-Valevski A,Kesler A,Sela BA etal.Outbreak of life threatening thiamine deficiency in infants in Israel caused by a defective soy-based formula.Paediatrics 2005;115:e233-e238.

8. Firdous U, Sharara HA, Nahia FA, Al Saqqa M, Musaed S. Wernicke's encephalopathy and Hyperemesis gravidarum. Int $J$ Nutr Pharmacol Neurol Dis .2013;3:142-5

9. Harper CG,Giles M,Finlay-Jones R.Clinical signs in Wernicke Korsakoff complex:a retrospective analysis of 131 cases diagnosed at necropsy.J Neurol Neurosurg Psychiatry 1986;49:341-345.

10.Kantor S, Prakash S, Chandwani J, Gokhale A, Sarma K, Albahrani MJ.Wernicke's encephalopathy following hyperemesis gravidarum.Indian J Crit Care Med. 2014 March; 18(3): 164-166.

11.Lu J,Frank EL.Rapid HPLC measurement of thiamine and its phosphate esters in whole blood.Clin Chem 2008;54:901-906.

12.Ohnishi A, Tsuji S, Takamori $\mathrm{M}$, et al. Beriberi neuropathy. Morphometric study of sural nerve. J Neurol Sci.1980;45:177-90.

13.Otsuka F,Tada K,Ogura T,Hayakawa N,Mimura Y,Yamauchi T,Inoue N,Making H,Kudo T.Gestational thyrotoxicosis manifesting as Wernicke Encephalopathy- A Case Report. Endocrine Journal 1997, 44(3), 447-452.

14.Reuler JB,girard DE,Cooney TG.Current concepts.Wernicke's encephalopathy.N Engl J Med 1985;312:1035-1039.

15.Sechi G,Serra A.Wernicke's encephalopathy:new clinical settings and recent advances in diagnosis and management.Lancet Neurol 2007;6:442-455.

16.Sulaiman W, Othman A, MohamadM,Salleh HR, Mushahar L.Wernicke's Encephalopthy Associated with Hyperemesis Gravidarum -
A Case Report.Malays J Med Sci.2002 July; 9(2): 43-46.

17.Thomson AD,Marshall EJ.The natural history and pathophysiology of Wernicke's encephalopathy and Korsakoff's psychosis Alcohol and Alcoholism.2006; 41 (2): 151158.

18.Togay-Isikay C, Yigit A, Mutluer N. Wernicke's encephalopathy due to hyperemesis gravidarum and underrecognised condition. Aust N Z J Obstet Gynaecol 2001; 41: 453-6.

19.Victor M Adams RD Collins GH.The Wernicke-Korsakoff Syndrome and Related Neurologic Disorders Doe to Alcoholism and Malnutrition.2 ${ }^{\text {nd }} \quad$ ed.Philadelphia,PA:FA Davis;1989.

20.Wang S, Hou X, Ding S, Guan Y, Zhen H, Tu $\mathrm{L}$, et al. Refractory hypotension in a patient with Wernicke's encephalopathy. Alcohol. 2012;47:48-51.

21.Yorek MA, Wiese TJ, Conner CE, et al. Reduced $\mathrm{Na}+\mathrm{K}+$ adenosine triphosphatase activity and motor nerve conduction velocity in l-fucose-fed rats is reversible after dietary normalization. Metabolism 1996;45:229-34.

22.Yoon CK, Chang MH, Lee DC. WernickeKorsakoff Syndrome Associated with Hyperemesis Gravidarum .Korean Journal of Ophthalmology 19(3):239-242, 2005.

23.Zuccoli G,Pipitone N.Neuroimaging findings in acute Wernicke's encephalopathy:review of literature. $A J R A m J$ Roentgenol 2009;192:501-508.

Parul Dubey, Sachin Narvekar, Deepa S.Nadkarni and Shradha Naik (2016), International Journal of Case Reports in Medicine, DOI: 10.5171/2016.376298 